\title{
Internal-noise-enhanced signal transduction in neuronal systems
}

\author{
Wei Wang ${ }^{1}$ and Z. D. Wang ${ }^{2}$ \\ ${ }^{1}$ Department of Physics and Institute for Solid State Physics, Nanjing University, Nanjing 210093, People's Republic of China \\ ${ }^{2}$ Department of Physics, The University of Hong Kong, Pokfulam Road, Hong Kong
}

(Received 18 September 1996)

\begin{abstract}
The ability of a neuron to detect and enhance a weak periodic flow of information within an "internalnoise" background has been studied through the mechanism of stochastic resonance. Two kinds of nonlinear synaptic input, a coherent firing of spikes from a number of coupled neurons and an irregular firing of spikes from a single neuron, are considered as the internal noise for a neuron. The output signal-to-noise ratio (SNR) is found to be finite. This nonzero SNR is able to account for the relevant experiments where the SNR is nonzero when the external noise is switched to zero. [S1063-651X(97)12705-2]

PACS number(s): 87.10.+e, 05.40. $+\mathrm{j}$
\end{abstract}

\section{INTRODUCTION}

Recently, there has been considerable interest in the studies of stochastic resonance (SR) by which a small periodic signal forcing nonlinear system can be amplified by the addition of a stochastic force, or external noise, to the signal $[1,2]$. SR can optimize the output signal-to-noise ratio (SNR); the SNR first increases to a maximum and then decreases as the intensity of external noise increases $[3,4]$. So far, SR has been demonstrated in a variety of physical experiments ranging from ring lasers, various solid state devices including superconducting quantum interference devices, to noise-driven chaotic attractors. Among many applications, it has been shown that neurons can detect and transmit a weak information signal in a sensory neural system via the SR since the neurons have a typical bistate feature, a firing or nonfiring state. Some experiments on the neural systems have been reported to show such nonlinear phenomena [5]. The earliest experiment was done by Douglass et al. [5]. They used near-field mechanoreceptors, located on the crayfish tailfan, in which small motions of cuticular hairs are transduced by their associated sensory neurons into spikes that propagate along the sensory nerves. Their experiment shows very clearly that weak signals can be enhanced by an optimal level of external noise in single sensory neurons. However, in this experiment and the others a nonzero SNR is found when the external noise is switched to zero [5]. This is believed to be due to the existence of internal noise in the neuronal systems.

Theoretically, there have been some studies on SR for neural systems from models like the integrate-and-fire model by Bulsara et al. [6], and Fitzhugh-Nagumo equations by Wiesenfeld et al. [3] and Collins et al. [4,7]. In all of these models, the controlling variable for characterizing the SR is external noise. Although in some cases the coupling between neurons within the system was also considered, the importance and effect of the internal noise, especially the noiselike synaptic input from neurons, have not been elucidated in detail. From neurobiology, we know neuronal activity is noisy, irregular, or aperiodic [8], which results from either the nonlinear dynamical behavior of the neuron itself $[9,10]$ or the external input from the ambient noise and the internal input from other neurons within the system. There are nu- merous internal sources of noise, some of which could be listed as follows [8,9]: (1) effects of the activities of adjacent neurons; (2) external input variability; (3) fluctuations of postsynaptic uptake efficiency; (4) fluctuations of dendritic or soma membrane parameters; (5) fluctuations in the threshold constants for neuron firing; (6) variations in the number of transmitter molecules at acceptor sites, etc. Among them, except the external input, the most important one might be the first item in the list, i.e., the synaptic input (or synaptic current) from other neurons via excitatory or inhibitory interaction. Generally, a total synaptic current on a neuron produced by both kinds of interactions is aperiodic and noiselike. We call this synaptic current the internal noise. Thus, by this consideration we propose the following question, which is addressed in this work. The question is whether the neuron can have the ability to detect a weak information signal through the mechanism of SR when a neuron is situated at both a signal input and an "internal noise," the aperiodic firing background. If this is true, the neurons will need no addition of external noise to detect an information signal in some cases, and the aperiodic background will play the role of external noise, as in the cases that have been well studied [1-5].

In this work, we show that a neuron can detect a weak information flow, a periodic input, within an "internal noise"' background of either aperiodic firing of a single neuron or coherent firing of a coupled neuronal network. This "internal noise" assists the occurrence of SR, and enables us to recover the experimental results of the nonzero SNR in the absence of the external noise [5]. The outline of this paper is as follows. In Sec. II we describe the model of a neuron detecting a weak periodic signal within an internal noise background. In Sec. III, we present and discuss the results. In Sec. IV a conclusion is given.

\section{MODEL}

We consider that a working neuron ( $W$ neuron) obtains a weak periodic input, the information input, and a synaptic input from other neurons, the background neurons ( $B$ neurons). We take all neurons as the well-established modified Fitzhugh's neuron model, the Hindmarsh-Rose neuron model [11]. The system is constructed by the following equations: 


$$
\begin{gathered}
\frac{d X_{W}}{d t}=Y_{W}-a X_{W}^{3}+b X_{W}^{2}-Z_{W}+I_{s}+\frac{1}{N} \sum_{i=1}^{N} J_{i B} S_{i B}(t) \\
\frac{d Y_{W}}{d t}=c-d X_{W}^{2}-Y_{W} \\
\frac{d Z_{W}}{d t}=r\left[s\left(X_{W}-X_{0}\right)-Z_{W}\right] \\
\frac{d X_{i B}}{d t}=Y_{i B}-a X_{i B}^{3}+b X_{i B}^{2}-Z_{i B}+I_{i}^{e}+\frac{1}{N} \sum_{j=1, j \neq i}^{N} J_{i j} S_{j}(t) \\
\frac{d Y_{i B}}{d t}=c-d X_{i B}^{2}-Y_{i B} \\
\frac{d Z_{i B}}{d t}=r\left[s\left(X_{i B}-X_{0}\right)-Z_{i B}\right]
\end{gathered}
$$

where all parameters are held constant at $a=1.0$, $b=3.0, \quad c=1.0, d=5.0, s=4.0, \quad r=0.006, \quad$ and $\quad X_{0}=$ -1.6 . Each neuron is characterized by three time-dependent variables: the membrane potential $X$, the recovery variable $Y$, and a slow adaptation current $Z$. The external information input for the $W$ neuron is a subthreshold periodic signal $I_{s}=A_{1} \sin 2 \pi f t$ in Eq. (1). The $W$ neuron also receives an "internal noise" synaptic input $J_{i B} S_{i B}(t)$ from the $i$ th $B$ neuron when the $i$ th $B$ neuron is active, i.e., $S_{i B}(t)=\theta\left(X_{i B}(t)-X^{*}\right)$ with $X^{*}$ being a threshold value of membrane potential and $\theta(x)=1$ if $x \geqslant 0$ and $\theta(x)=0$ if $x<0$. The coupling strength between the $W$ neuron and the $i$ th $B$ neuron is $J_{i B}$, which is a constant or distributed depending on the model described below. The $B$ neurons are assumed to be coupled themselves by an interaction $J_{i j} S_{j}(t)$, i.e., when the $j$ th $B$ neuron is fired, it will have a synaptic interaction on the $i$ th $B$ neuron through a coupling strength $J_{i j}$. These background neurons are situated in a stimulus-induced oscillatory state; i.e., $I_{i}^{e}$ is a constant. The dynamics of the system is controlled by the coupling strength $J_{i B}$ and the stimulus $I_{i}^{e}$. The simulations are done by using a modified fourth-order Runge-Kutta method, and the time scale is based on the neurobiological results [12].

\section{RESULTS AND DISCUSSION}

\section{A. Single neuron background}

First, we consider a system with only one $B$ neuron, and we take the external stimulus $I^{e}=3.0$ with which the $B$ neuron has a chaotic output [13]. The firing of spikes of the $W$ neuron has been converted into a time series of standard pulses $V(t)$ with $V_{1}=1.0$ within a wide $\Delta \tau=2 \mathrm{~ms}$ and $V_{0}=0$ related to the firing and nonfiring state, respectively. This time series is then transferred into a power spectrum through the fast Fourier transform. The final result is obtained by taking an average over 38 spectra within a total duration of approximately $1.3 \mathrm{~min}$. Figure 1 shows one example of the stochastic resonance of the $W$ neuron. The spectrum of output of the $B$ neuron [see Fig. 1(a)], used as input for the $W$ neuron, is not periodic, but has a main peak around

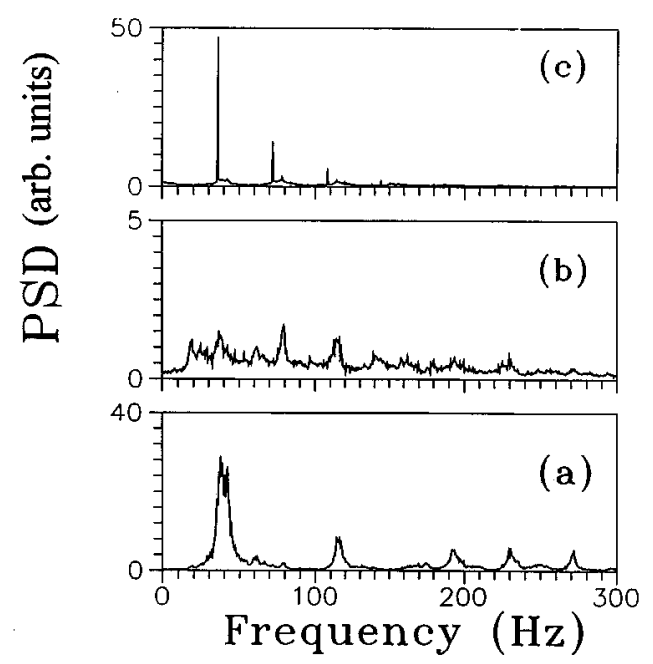

FIG. 1. The power spectrum density (PSD) of a $W$ neuron detecting a periodic information signal $I_{s}=A_{1} \sin 2 \pi f t$ with $A_{1}=0.42$, which is slightly lower than the threshold value of $A_{1}=0.44, f=36 \mathrm{~Hz}$. The $W$ neuron is situated at a single $B$ neuron with a stimulus $I^{e}=3$ and a coupling strength between these two neurons $J=3.5$. The vertical axis is in arbitrary units. (a) The synaptic input to the $W$ neuron from the output of a single $B$ neuron; (b) the output of the $W$ neuron in the absence of the information signal; (c) the output of the $W$ neuron in the presence of the information signal.

$40 \mathrm{~Hz}$ that related to the deterministic nonlinear firing of spikes [14]. In the absence of the information signal, this internal deterministic noise only produces a broadband noise output for the $W$ neuron [see Fig. 1(b)]. Nevertheless, if the information signal is added, it is amplified by the $W$ neuron through the mechanism of stochastic resonance as shown in Fig. 1(c), while with the signal alone there is no output from the $W$ neuron since the threshold amplitude of the signal is $A_{1}=0.44$. In Fig. 1(c), there is obviously a peak at the signal frequency $f=36 \mathrm{~Hz}$ and its harmonics. That is, there are also peaks at the even harmonics. This is in agreement with all the experimental results [5] because the firing and nonfiring of the neuron are not symmetric as in the case studied by Zhou and Moss [15]. Clearly, the nonsymmetry of the firing and nonfiring states can be understood from nonsymmetric residence times' density functions $P_{f}(\tau)$ and $P_{\mathrm{nf}}(\tau)$ related to the firing and nonfiring, respectively. The neuron spends more time in the nonfiring state. On the contrary, these two functions are symmetric in the case of the double-well potential [15].

The SNR is defined by $\mathrm{SNR}=10 \log _{10}(S / B)$ where $S$ and $B$ represent the values of the output power spectrum density (PSD) at the peak and the base of the signal feature, respectively [16]. The SNR against the coupling strength $J_{i B}=J$ is plotted in Fig. 2, which shows an optimized coupling strength with which the SNR has a maximum around the coupling strength $J_{i B}=2.0$. When $J_{i B}>6$, the deterministic noise background is very strong and there are many peaks in the spectrum around the frequency of the signal; the peak at the signal is small and is hard to define. But the detecting ability of the $W$ neuron is decreased within the strong aperiodic background. The interspike interval (ISI) histogram is 


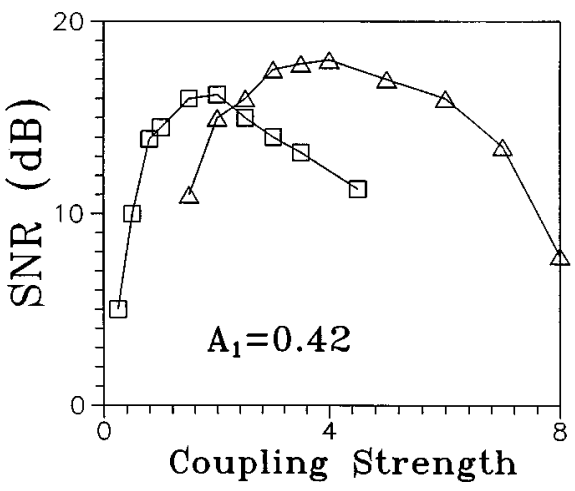

FIG. 2. The signal-to-noise ratio (SNR) against the coupling strength. The synaptic input from: (a) a single neuron background with $I^{e}=3$ (open square); (b) 500 coupled neurons background with the stimuli on the $B$ neurons distributed in a range of $I_{i}^{e} \in[0,5]$ (open triangle).

shown in Fig. 3. The peaks are located at integer multiples of the period of input signal $T=28 \mathrm{~ms}$ up to 10 periods, and an approximately exponential decaying of the heights of the peaks can also be seen in the inset in Fig. 3. We can also define a return map, a scatter plot of the interval $\delta_{i+1}$ versus $\delta_{i}$, with $\delta_{i}$ being the interval between the $i$ th firing and $(i-1)$ th firing (not shown). We have seen that the dots form a lattice with intersections at integer multiples of the period $T_{s}$ of the interspike intervals, and a periodically modulated firing of spikes of the $W$ neuron. These results are in agreement with the experiments and numerical simulation (see Ref. [17], and references therein).

\section{B. Multineuron background}

Next we take a number of coupled neurons as the "internal noise" background, say, $N=500$; i.e., 500 neurons coupled together contribute a coherent oscillatory noise background to the $W$ neuron. In order to model a more realistic situation of the neural system, we consider the global interaction strength $J_{i j}$ to be not only excitatory with $J_{i j}>0$, but also inhibitory with $J_{i j}<0$. Actually, for the ex-

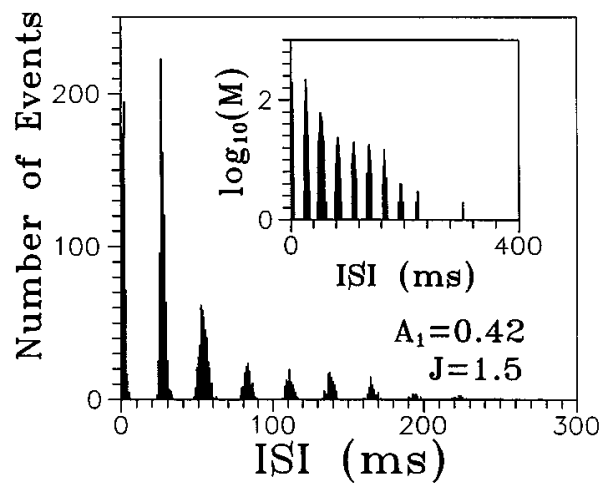

FIG. 3. The ISI histogram of a $W$ neuron with a synaptic input from a single neuron background. The stimulus for the $B$ neuron is $I^{e}=3$ and the coupling strength equals $J=1.5$ between the $W$ neuron and the $B$ neuron. The inset shows the logarithmic numbers of events $(M)$ vs the ISI.

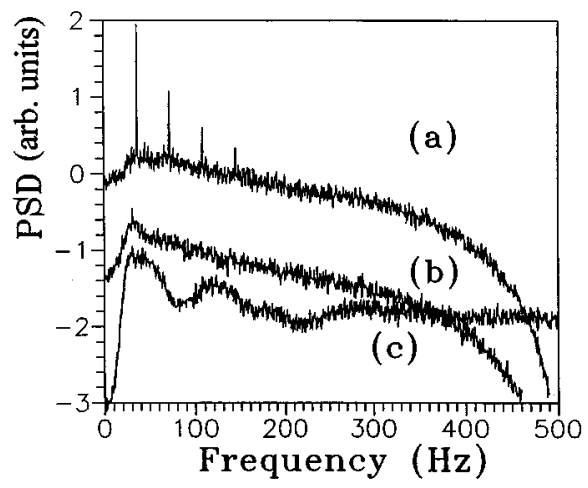

FIG. 4. The power spectrum density (PSD) of the output of a $W$ neuron situated in a multineuron background of 500 coupled neurons with a stimulus $I_{i}^{e}=3$ and the coupling strength $J_{i j}$ distributed in a range of $[-1,10]$. The vertical axis is logarithmic in arbitrary units. (a) The output with information input $I_{s}=A_{1} \sin 2 \pi f t$ with $f=36 \mathrm{~Hz}, A_{1}=0.42$; (b) the output without information input. The vertical value has been shifted with -1 ; (c) the background input of a network of 500 coupled neurons to the $W$ neuron. The vertical value has been shifted with -2 .

citatory coupling, there is a positive excitatory postsynaptic potential (EPSP), while for the inhibitory coupling there is a negative inhibitory postsynaptic potential (IPSP). The spatial and temporal summation of these synaptic potentials, EPSP and IPSP, on a local neuron will result in firing or nonfiring of spikes, which makes the neuron form a function. Thus, the coupling strength $J_{i j}$ is assumed to be distributed in some ranges, and its value is positive or negative at random. For simplicity, we set $J_{i B}$ as a coupling between the $i$ th and the $W$ neuron (first) with $i=2,3, \ldots, N$. In the meantime, the stimulus is kept as $I_{i}^{e}=3.0$ for all the neurons.

By making these considerations, we have performed numerical simulations as we did for the single neuron background. Figure 4 shows the stochastic resonance of the $W$ neuron and the internal noise background. We can see clearly that when the input signal is switched on there are some peaks at the frequency of the signal and its harmonics, and the first peak is about two orders of magnitude high riding on the background [curve (a)]. The internal noise background shows a broadband spectrum but there is a broad peak around $40 \mathrm{~Hz}$ [curve (c)]. We have performed simulations for different distributions of the coupling strength $J_{i j}$ and different values of the constant stimuli $I_{i}^{e}$. We found that all of them show the same feature of stochastic resonance. But, the height of the main peak of the resonance is influenced by the value of the stimulus and the forms of the distributed coupling strength. Especially, when the percentage of the inhibitory coupling $\eta$ increases, the main peak decreases. For example, when $\eta>75 \%$, the main peak due to the input signal will disappear. However, with these different percentages, or different ratios between two kinds of couplings, the dynamics of the background itself is very complicated. We will discuss this elsewhere.

For comparison with the result of the multineuron background and that of the single-neuron background, we choose another procedure; we assume the stimuli to be randomly distributed in some range, say, $I_{i}^{e} \in[0,5]$, and keep the cou- 
pling strength to be the same value for all neurons, $J_{i j}=J$. Numerical simulation shows that the system exhibits the same phenomenon of stochastic resonance as that of the distributed coupling strength shown in Fig. 4. The SNR is plotted in Fig. 2 with triangles. One can see that the SNR is slightly higher than that of the single-neuron background, and the peak is shifted to a higher coupling strength about $J=4.0$. For a coupling strength $J>2.5$ the multineuron background makes the $W$ neuron produce higher SNR than that of the single-neuron background; while for $J<2.5$ the $W$ neuron has high SNR for the case of the single-neuron background. Obviously, this behavior results from the coherent oscillation between the neurons in the case of the multineuron background. When the coupling strength is small, the $B$ neurons have a weak coherent oscillation since some neurons have stimuli $I_{i}^{e}$ that are not in the nonlinear region of $2.9<I_{i}^{e}<3.4$ [13]. That is, some neurons will behave with the periodic firing of spikes, and the background shows a weak synchronized chaos $[13,18]$. Therefore the SNR will be small.

As a result of the stimulations shown above, we see that the SNR is nonzero in a large range of values of the coupling strength and different stimuli for the $B$ neurons in both cases of the deterministic internal noise background. This gives an explanation for the experimental measurements that when the external noise switches to zero the SNR still stands [5]. In Ref. [19], Douglass and Moss did an experimental study on the effect of the internal noise for the SNR. They found that by changing the acclimated temperature for the sample there is a convex SNR versus the temperature. But, when they plotted the SNR against the internal noise, they found a monotonic increase in SNR with the noise intensity and, specifically, no maximum at an optimum noise intensity. They concluded that the convex SNR versus the temperature cannot be interpreted as SR. As a matter of fact, this may result from the fact that there is a decrease in the internal noise itself after it reaches a maximum as the temperature increases. In order to see that our results shown in Fig. 2 come from SR, we have calculated the average of the total internal noise input $\left\langle\xi_{2}\right\rangle$ and its variance $\sigma^{2}=\left\langle\xi_{2}^{2}\right\rangle-\left\langle\xi_{2}\right\rangle^{2}$ with $\xi_{2}(t)=N^{-1} \sum_{i=1}^{N} J_{i B} S_{i B}(t)=N^{-1} J \sum_{i=1}^{N} S_{i B}(t)$ as a function of the coupling strength $J$. In Fig. 5 we show the results. We can see that there is a linear increase in the averaged noise $\left\langle\xi_{2}\right\rangle$ and a cubic increase in the variance $\sigma^{2}$ as the coupling strength increases. In other words, the SNR will show a maximum if we plot it against $\left\langle\xi_{2}\right\rangle$ or $\sigma^{2}$. Therefore, we can conclude that the SNR obtained in this work is a result of the SR, and our simulations show that the internal noise, a coherently cooperative oscillation, or a noncoherent chaotic firing of spikes will cause the SR. As the number of neurons in the background increases, on one hand, the background supports a coherent oscillation to the $W$ neuron; the SNR peak will shift to the region of high coupling strength on the other hand. But, 500 neurons is large enough for modeling a real case, and we have seen almost no difference for a simulation with 1000 neurons.

In Fig. 6(a), we show the interspike interval (ISI) histogram of a system of 500 coupled neurons with coupling strength $J_{i j}=4$ for all the neurons and distributed stimuli $I_{i}^{e} \in[0,5]$. The peaks are located at the integer multiples of

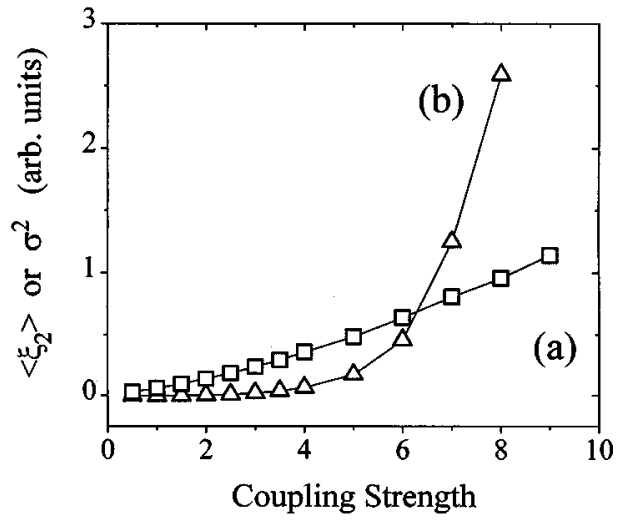

FIG. 5. The average $\left\langle\xi_{2}\right\rangle$ and the variance $\sigma^{2}=\left\langle\xi_{2}^{2}\right\rangle-\left\langle\xi_{2}\right\rangle^{2}$ of the synaptic input $\xi_{2}(t)=N^{-1} J \sum_{i=1}^{N} S_{i B}(t)$ vs the coupling strength $J$ in the multineuron background of 500 coupled neurons with coupling strength $J$ and stimulated by $I_{i}^{e}$ distributed in a range of [0,5]. (a) $\left\langle\xi_{2}\right\rangle$ vs $J$ (open square); (b) $\sigma^{2}$ vs $J$ (open triangle).

the period of input signal $T_{s}=25 \mathrm{~ms}$ up to 6 periods, and there is an approximately exponential decaying of the heights of peaks. The first peak (at $n T_{s}$ with $n=0$ ) is due to a recording procedure (ISI shorter than 2 ms should be omitted), which will be neglected in the following discussion. From the inset in Fig. 6(a), we can also see that the return map consists of a lattice with intersections at integer multiples of the signal period. The firing of the $W$ neuron is modulated by the periodic signal, but it is basically concentrated within the first period $(n=1)$; i.e., the number of firing events takes up to more than $80 \%$ for this peak. Actually, this dominant peak relates to the maximum of SNR. This indicates that the firing of the $W$ neuron is almost in synchrony with the signal. Physically, it represents a resonance of the signal with the noise background in the system.

In Figs. 6(b) and 6(c), we show the ISI histogram of a $W$ neuron in a background of 500 coupled neurons with a distributed coupling strength $J_{i j} \in[-1,10]$ and stimuli $I_{i}^{e}=3$ for the system in the absence and presence of the input signal. In the absence of the input signal, $A_{1}=0$, the ISI shows a broad peak around $30 \mathrm{~ms}$. This is due to the synaptic input, which is a coherent oscillation with a broadband of low frequency [see curve $(c)$ in Fig. 4]. The return map shows a wide distribution of points at $30 \mathrm{~ms}$ [see Fig. 6(b)]. However, the situation is different in the presence of the periodic signal. The ISI has two narrow peaks at 25 and 50 ms, respectively [see Fig. 6(c)]. The first peak has about 200 spikes, which is larger than that of the first peak in Fig. 6(b). The return map also shows clusters at the intersections of the multiples of the period of the periodic signal. From these two plots, Figs. 6(b) and 6(c), we may conclude that the firing of spikes of the $W$ neuron could be modulated both by the periodic signal and by the coherent oscillation in the background itself, but the modulation is stronger for the former than for the latter one. Thus, it is reasonable that when the weak information signal is switched off, the spectrum of the output is a noiselike background with a small peak about 2 $\mathrm{dB}$ height around $\omega=32 \mathrm{~Hz}$ [see curve (b) in Fig. 4]. We 

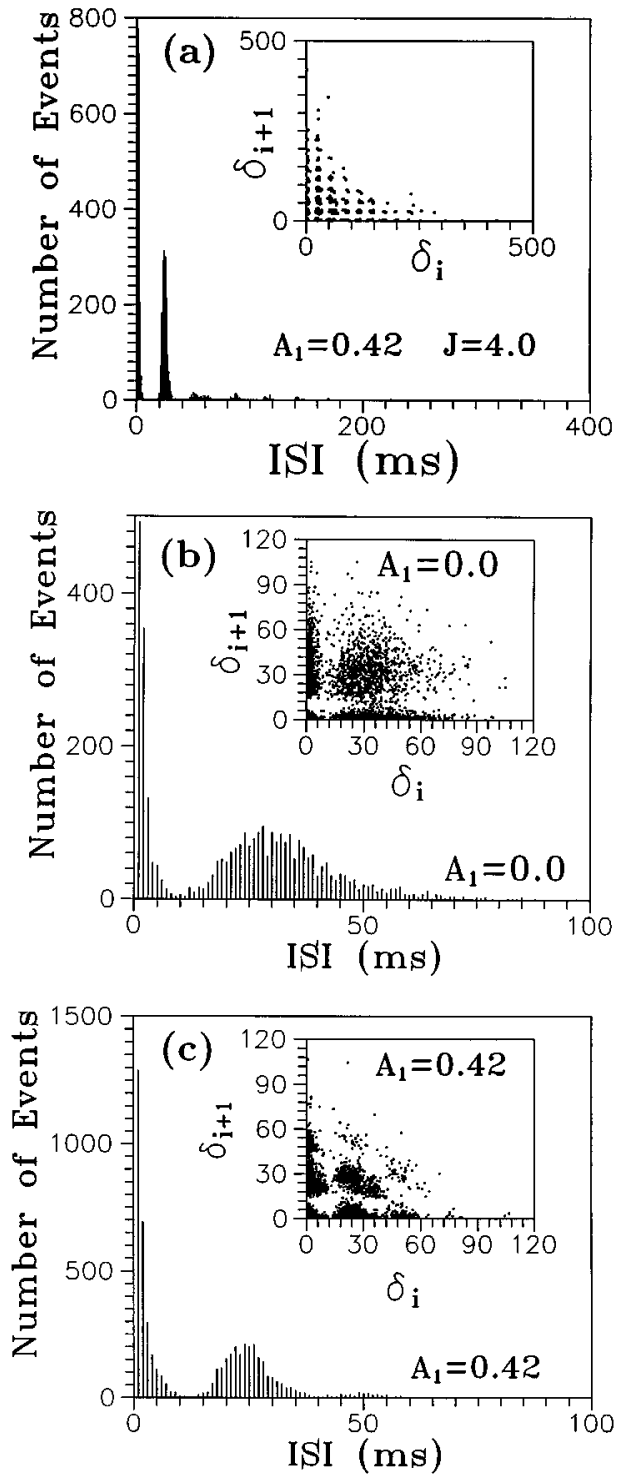

FIG. 6. The ISI histogram of a $W$ neuron situated in a multineuron background of 500 neurons with (a) a coupling strength $J_{i j}=4$ and a distributed stimuli $I_{i}^{e} \in[0,5]$; (b) a stimulus $I_{i}^{e}=3$ and distributed coupling strength $J_{i j} \in[-1,10]$ in the absence of the input signal; (c) the same as (b) but with the input signal. The insets show the first return map, respectively.

have checked that if the number of $W$ neurons is increased, this peak will also increase slightly. That is, a network may transfer the coherent oscillation or the stimulation information itself. We plan to report on this in detail elsewhere.

Finally, let us make a remark on our model and result. The internal noise considered in this work is due to the nonlinear synaptic input from other neurons in the neural system. It is controlled by the coupling strength or the stimulus. Our motivation is to model the realistic situation of the neurons from a mechanism as follows. In experiments, the sample is acclimated at different temperatures (see Douglass et al. in Ref. [5]), or the sample is situated at different thermal stimuli [20]. This may enable the neurons to form different synaptic strengths on other neurons, which makes a change in the internal noise, while for the case of thermal stimulus, the internal noise is a direct result of the firing of neurons, which are induced by the stimulus. Although for the real neurons the internal noise is a cooperative effect of various sources and it may have a complicated connection with a number of parameters, such as the coupling strength, stimulus, and so on, our work presents a significant modeling result and provides an interpretation for the experiments. Actually, Eq. (1) could be presented as

$$
\frac{d X}{d t}=f(X, Y, Z)+A_{1} \sin 2 \pi f t+a_{1} \xi_{1}(t)+a_{2} \xi_{2}(t),
$$

where $\xi_{1}(t)$ is the external noise added to the system and $\xi_{2}(t)$ is the internal noise. When the external noise is strong, the effect of the internal noise could be eliminated, and vice versa. Generally, the external noise is taken as a Gaussian white noise. A mixed noise from two terms will also behave basically as white noise when the intensity of external noise is larger than, or approximately equal to that of the internal noise. Thus, the SNR will show a maximum that results from the SR as the intensity of the external noise increases. However, whether there is a maximum in the figure of SNR against the intensity depends on the level of the internal noise. In the model Eq. (1) or Eq. (7), the internal noise is independent of the external input for the $W$ neuron; it only depends on the dynamics of the coupling neurons in the background. It is quite possible that the internal noise is large enough compared with the external noise in some cases. Under these circumstances, the maximum may be exceeded due to the internal noise and the SNR only shows a decrease when the external noise increases from zero. Indeed, the experimental measurements show such a behavior [19].

From the discussion above, we know that there is a competition between the external noise and the internal noise, and which noise dominates the influence depends on the intensities of them. One important argument is that the living animal can use internal noise to perform information detecting (if the information detecting process works through the mechanism of stochastic resonance). For example, if any one animal is placed in a quiet environment without any external disturbance, experiments show that he can respond to the external information more sensitively.

\section{CONCLUSION}

In conclusion, our results show that the neuron can detect a weak information signal in the absence of the external noise input. A deterministic chaotic firing (coherent or noncoherent firing between neurons), a kind of "internal noise," can play the role of the external noise. By such internal synaptic input a neuron behaves as stochastic resonance and shows a characteristic peak at the frequency of the input signal. But the coherent oscillatory background may shift the optimized peak of SNR to the high coupling region, which makes the neuron fire more synchronously $[13,18]$. As a result, the information processing or computation may be represented or coded easier through such dynamical behavior [4-7,21]. Our results come to one point: the SR process still works even when the external noise is zero. This may explain the recent experimental measurements on the real neurons. 
[1] Proceedings of the NATO Advanced Research Workshop on Stochastic Resonance in Physics and Biology, edited by F. Moss, A. Bulsara, and M. F. Shlesinger [J. Stat. Phys. 70 (1993)].

[2] Proceedings of the International Workshop on Fluctuations in Physics and Biology: Stochastic Resonance, Signal Processing and Related Phenomena, edited by R. Mannella and P. V. E. McClintock [Nuovo Cimento Soc. Ital. Fis. 17 (1995)].

[3] R. Benzi et al., Tellus 34, 10 (1982); L. Gammaitoni et al., Phys. Rev. Lett. 62, 349 (1989); B. McNamara et al., Phys. Rev. A 39, 4854 (1989); P. Jung et al., Europhys. Lett. 8, 505 (1989); G. Hu et al., Phys. Rev. A 42, 2030 (1990); A. R. Bulsara et al., Phys. Rev. E 47, 3734 (1993); K. Wiesenfeld et al., Phys. Rev. Lett. 72, 2125 (1994).

[4] A. Bulsara, E. W. Jacobs, T. Zhou, F. Moss, and L. Kiss, J. Theor. Biol. 152, 531 (1991); A. Bulsara et al., Phys. Rev. E 53, 3958 (1996); A. Longtin, A. R. Bulsara, and F. Moss, Phys. Rev. Lett. 67, 656 (1991); J. J. Collins, C. C. Chow, and T. T. Imhoff, Nature 376, 236 (1995).

[5] J. K. Douglass, L. Wilkens, E. Pantazelou, and F. Moss, Nature 365, 337 (1993); K. Wiesenfeld and F. Moss, ibid. 373, 33 (1995); S. M. Bezrukov and I. Vodyanoy, ibid. 378, 362 (1995); J. E. Levin and J. P. Miller, ibid. 380, 165 (1995).

[6] A. R. Bulsara, S. B. Lowen, and C. D. Rees, Phys. Rev. E 49, 4989 (1994); A. R. Bulsara and G. Schmera, ibid. 47, 3734 (1993); M. E. Inchiosa and A. R. Bulsara, ibid. 52, 327 (1995); F. Chapeau-Blondeau, ibid. 53, 5469 (1996); F. ChapeauBlondeau, X. Godivier, and N. Chambet, ibid. 53, 1273 (1996).

[7] J. J. Collins, C. C. Chow, and T. T. Imhoff, Phys. Rev. E 52, R3321 (1995).
[8] H. C. Tuchwell, Stochastic Processes in the Neuronscience (SIAM, Philadelphia, 1989); A. V. Holden, Models of the Stochastic Activity of Neurons, Lecture Notes in Biomathematics Vol. 12 (Springer, Berlin, 1976); G. Sampath and S. K. Srinivasan, Stochastic Models for Spike Trains of Single Neurons, Lecture Notes in Biomathematics Vol. 16 (Springer, Berlin, 1977).

[9] J. G. Taylor, in Neurodynamics, edited by F. Pasemann and H. D. Doebner (World Scientific, Singapore, 1991).

[10] Wei Wang, J. Phys. A. 22, L627 (1989); Commun. Theor. Phys. 17, 93 (1992); 18, 265 (1992).

[11] J. L. Hindmarsh and R. M. Rose, Nature 296, 162 (1982); Proc. R. Soc. London B 221, 87 (1984); 225, 161 (1985).

[12] For the HR's neuron, the time scale is defined as 10 units equaling $2 \mathrm{~ms}$, the width of the action potential, which is related to the experimental results.

[13] Wei Wang, G. Perez, and H. A. Cerdeira, Phys. Rev. E 47, 2893 (1993).

[14] Wei Wang and Z. D. Wang, Phys. Rev. Lett. (1996).

[15] T. Zhou and F. Moss, Phys. Rev. A 41, 4255 (1990).

[16] A. R. Bulsara and L. Gammaitoni, Phys. Today 49(3), 39 (1996).

[17] A. Longtin, A. Bulsara, D. Pierson, and F. Moss, Biol. Cybern. 70, 569 (1994)

[18] D. Hansel and H. Sompolinsky, Phys. Rev. Lett. 68, 718 (1992).

[19] J. K. Douglass and F. Moss, Naval Research Reviews XLV, 30 (1993).

[20] H. A. Braun, H. Wissing, K. Schafer, and M. C. Hirsch, Nature 367, 270 (1994).

[21] J. J. Hopfield, Nature 376, 33 (1995). 